\title{
Comment on "Stiffness degree of ankle range of motion in diabetic patients with atypical amputation"
}

\author{
Minjie Jiang ${ }^{1}\left(\mathbb{D},{\text { Chenchen } \operatorname{Pan}^{1} \mathbb{D} \text {, Lianping He}}^{1 *}\right.$
}

\section{Dear Editor,}

We are more than honored to read the high-level articles published by Simón-Pérez et al. ${ }^{1}$ Their study found that feet with atypical distal amputations feature the classification of dorsal flexing movement in the ankle lower than the contralateral foot. However, as far as I am concerned, there are still some issues worth discussing with the author.

First, the author did not explain what statistical methods were used in the method section. We do not know what the p-value is which indicates that the result is significant. In addition, both type 1 and type 2 diabetes were included in the sample. We think that author should further discuss in-depth the effects of two different types of diabetes on the stiffness of ankle joints. And the sample size of the patients with diabetes 1 was too small.

Second, the author collected the general demographic characteristics of the sample in the study. This is worth approving. However, the author should then analyze the relativity between age, gender, weight, size, and body mass index (BMI) and stiffness degree of ankle joint activity in diabetic patients. In contrast, the author stated that they collected the general demographic characteristic information of size. But we do not find the corresponding information in Table 1 . Besides, the sample size of women patients was too small.

The author pointed out in the discussion section that age, smoking, BMI, and the level of Hb1Ac did not affect the classification of ankle stiffness degree. We are confused about how they came to this conclusion. The author did not make relevant statistical analysis in this article, and we cannot even see the corresponding $\mathrm{p}$-value.

Finally, in the study, the conclusion is also confusing, which seems to be contrary to the research goal of this article. Foot Posture Index (FPI) was a specific value rather than a tendency. The author stated that FPI reflects the stiffness degree of ankle joint in the patients with diabetes.

\section{AUTHORS' CONTRIBUTIONS}

MJ: formal analysis, writing - original draft, and data curation. CP: conceptualization and writing - review and editing. LH: Conceptualization, investigation, funding acquisition, methodology, project administration, resources software, supervision, validation, visualization, writing - review and editing.

\section{REFERENCE}

1. Simón-Pérez E, Simón-Pérez C, Alonso-Peña D, Pontón-Cortina A, Chicharro-Luna E, Martínez-Nova A, et al. Stiffness degree of ankle range of motion in diabetic patients with atypical amputation. Rev Assoc Med Bras. 2020;66(2):216-21. https://doi.org/10.1590/1806-9282.66.2.216 